\title{
DIFFUSION THEORY CAN BE APPLIED TO ANTIBODIES ATTACHING TO LIGAND SITES
}

\author{
D. P. WILSON ${ }^{1}$ and D. L. S. MCELWAIN 1
}

(Received 3 April, 2003; revised 1 November, 2004)

\begin{abstract}
Humoral immunity is that aspect of specific immunity that is mediated by B lymphocytes and involves the neutralising of disease-producing microorganisms, called pathogens, by means of antibodies attaching to the pathogen's binding sites. This inhibits the pathogen's entry into target cells. We present a master equation in both discrete and in continuous form for a ligand bound at $n$ sites becoming a ligand bound at $m$ sites in a given interaction time. To track the time-evolution of the antibody-ligand interaction, it is shown that the process is most easily treated classically and that in this case the master equation can be reduced to an equivalent one-dimensional diffusion equation. Thus well-known diffusion theory can be applied to antibody-ligand interactions. We consider three distinct cases depending on whether the probability of antibody binding compared to the probability of dissociation is relatively large, small or comparable, and numerical solutions are given.
\end{abstract}

\section{Introduction}

Antibodies bind to, and block, ligand sites on a pathogen (an infectious foreign agent) affecting its capacity to attach to target cell receptors. As a consequence, the ability of the pathogen to enter a target cell is inhibited. In addition, antigen-bound antibodies produce a signal that activates specific white blood cells, the macrophages, which then engulf and destroy the bound pathogen. Since viruses and many bacteria reproduce within cells, blocking the cell attachment would limit such pathogens from replicating. The time-dependent dissociation and recombination of complexes formed by antibodies attaching to the surface of ligands is a fundamental process in mathematical immunology in general, and in the study of humoral immunity, in particular, and is the topic of investigation in this paper. The aim here is to provide a novel way of estimating the time-evolution of the distribution of the specific number of bound antibodies (we track a cohort of pathogen-antibody aggregates).

\footnotetext{
${ }^{1}$ School of Mathematical Sciences, Queensland University of Technology, GPO Box 2434, Brisbane Qld 4001, Australia; e-mail: s.mcelwain@qut.edu.au.

(C) Australian Mathematical Society 2005, Serial-fee code 1446-1811/05
} 
Two approaches have been used to calculate the aggregate size distribution. The first approach, the obvious one, is to write down differential equations in the form of chemical rate equations for the concentrations of all possible ligand-receptor aggregates [3]. However, a complete description requires the solution of a large set of coupled ordinary differential equations [12], one for each aggregate. While this system is straightforward to formulate, the order of the system is very large. For example, rat basophilic leukemia cells have approximately $10^{5}$ receptors per cell and a chlamydial elementary body has approximately $2.86 \times 10^{4}$ receptors. If this approach is used to estimate a time-dependent aggregate distribution size the set of equations must be truncated $[3,14]$. A second approach is less general, but can be used to obtain the complete time-dependent aggregate size distribution by solving just two coupled nonlinear differential equations [11]. The kinetics of the ligand-receptor complexes distribution are presented in the form of a series $[1,10]$. Although this works well for relatively small numbers of binding sites (1-100), a simpler mathematical approximation would be very useful for a system when the number of binding sites is significantly greater [21]. Here we develop another approach to obtain the complete time-dependent aggregate size distribution for multivalent ligands (cell surfaces with many receptors) bound by molecules that bind at one receptor only. It involves solving a single diffusion equation. One example of such a binding molecule is the Fab fragment of an antibody, comprising one arm of the full Y-shaped antibody. While this restricts the model's applicability to antibody-pathogen interactions in general, there are many systems for which this assumption is appropriate. For example, the pent-valent adenovirus requires full occupancy by antibodies to achieve neutralisation. This can be achieved by Fabs but not by whole antibodies (IgG molecules in this case) [19]. A similar phenomenon has been found in antibody-Chlamydia interactions [20]. We also assume that all binding sites are equivalent and we assume that adsorbed particles do not interact, that is, the binding of a molecule at one site does not block the binding at a neighbouring site.

Our theory is similar to that used by Keck and Carrier [6], who investigated techniques for solving master equations for the coupled vibration-dissociation-recombination process for molecules and atoms.

\section{Modelling antibody attachment numbers on a pathogen}

Consider a pathogen bound at $n$ sites by antibodies. We assume that there is a probabilistically inferred rate at which the pathogen bound at $n$ sites can become a pathogen bound at $m$ sites. The discrete version of any such model is of the form

$$
\frac{\partial A(n, t)}{\partial t}=\sum_{m=0}^{N}[K(m, n) A(m, t)-K(n, m) A(n, t)],
$$


where $A(n, t)$ is the concentration of pathogens with $n$ antibodies attached and $N$ is the maximum number of antibodies that can be bound to a pathogen simultaneously. This equation states that pathogens bound by $n$ antibodies may leave this state by making transitions to pathogens bound by $m$ antibodies, gaining or losing antibodies, at a rate $K(n, m) A(n, t)$. We expect transitions from $n$ antibodies to $n-1$ or $n+1$ antibodies on a pathogen to dominate the rate function, $K$.

The discrete equation (2.1) for the dynamics of the pathogen-antibody concentrations has an analogous continuous version,

$$
\frac{\partial E(x, t)}{\partial t}=\int_{0}^{f} k\left(x^{\prime}, x\right) E\left(x^{\prime}, t\right)-k\left(x, x^{\prime}\right) E(x, t) d x^{\prime},
$$

where $k\left(x, x^{\prime}\right)$ is the probabilistically inferred rate of undergoing a transition from state $x$ to state $x^{\prime}$ per unit time and $f$ is the maximum number of antibodies on average that can attach to the surface of the pathogen simultaneously.

In the absence of immune clearance and cell infection the pathogen-antibody concentrations, $E(x, t)$, have a non-trivial equilibrium distribution, which we denote by $E_{e}(x)$. At equilibrium $\partial E(x, t) / \partial t$ is zero, and the requirement for detailed balancing [8] leads to the condition

$$
R\left(x^{\prime}, x\right)=k\left(x^{\prime}, x\right) E_{e}^{\prime}\left(x^{\prime}\right)=k\left(x, x^{\prime}\right) E_{e}(x)=R\left(x, x^{\prime}\right) .
$$

We use the equilibrium distribution to introduce the non-dimensionalised concentration

$$
X(x, t)=\frac{E(x, t)}{E_{e}(x)},
$$

which is the ratio of the concentration of pathogens with $x$ antibodies attached to the associated equilibrium concentration. Then (2.2) can be written in the symmetrical form

$$
E_{e}(x) \frac{\partial X(x, t)}{\partial t}=\int_{0}^{f} R\left(x, x^{\prime}\right)\left[X\left(x^{\prime}, t\right)-X(x, t)\right] d x^{\prime}
$$

\section{Transformation to the diffusion equation}

We now transform the master equation, (2.4), to an equivalent diffusion equation. The transformation assumes the integrand in (2.4) can be expanded in a Taylor series about $x^{\prime}=x$ and we assume that the kernel, $R\left(x, x^{\prime}\right)$, is separable, and is large only for $x^{\prime} \approx x$. We can then anticipate that the solution of (2.4) can be well approximated by the solution of

$$
E_{e}(x) \frac{\partial X(x, t)}{\partial t}=\int_{-\infty}^{\infty} R\left(x, x^{\prime}\right)\left[X\left(x^{\prime}, t\right)-X(x, t)\right] d x^{\prime} .
$$


Expanding $X\left(x^{\prime}, t\right)$ about $x^{\prime}=x$, we obtain the partial differential equation

$$
E_{e}(x) \frac{\partial X}{\partial t}=\mu_{1}(x) \frac{\partial X}{\partial x}+\frac{\mu_{2}(x)}{2} \frac{\partial^{2} X}{\partial x^{2}}+O\left(\mu_{3}\right),
$$

where

$$
\mu_{n}(x)=\int_{-\infty}^{\infty} R\left(x, x^{\prime}\right)\left(x^{\prime}-x\right)^{n} d x^{\prime}
$$

is the $n$th moment of the change in antibody level $\left(x^{\prime}-x\right)$ with respect to $R\left(x, x^{\prime}\right)$. Observing symmetry of $R\left(x, x^{\prime}\right)$ on interchange of $x$ and $x^{\prime}$ requires that

$$
R\left(x, x^{\prime}\right)=S(\bar{x},|\Delta|),
$$

where $\bar{x}=\left(x^{\prime}+x\right) / 2$ is the mean of the initial and final antibody levels and $\Delta=x^{\prime}-x$ is the change in the antibody levels. Assuming $S(\bar{x},|\Delta|)$ is sharply peaked at $\Delta=0$, we expand about $\Delta=0$ and obtain

$$
\begin{aligned}
\mu_{1}(x) & =\left.\int_{0}^{\infty} \frac{\partial S}{\partial \bar{x}}\right|_{\bar{x}=x} \Delta^{2} d \Delta+\left.\frac{2}{3 !} \int_{0}^{\infty} \frac{\partial S^{3}}{\partial \bar{x}^{3}}\right|_{\bar{x}=x} \Delta^{4} d \Delta+\cdots \\
& =\left.\int_{0}^{\infty} \frac{\partial S}{\partial \bar{x}}\right|_{\bar{x}=x} \Delta^{2} d \Delta+O\left(\Delta^{4}\right)
\end{aligned}
$$

and

$$
\begin{aligned}
\mu_{2}(x) & =2 \int_{0}^{\infty} S(x,|\Delta|) \Delta^{2} d \Delta+\left.\int_{0}^{\infty} \frac{\partial S^{2}}{\partial \bar{x}^{2}}\right|_{\bar{x}=x} \Delta^{4} d \Delta+\cdots \\
& =2 \int_{0}^{\infty} S(x,|\Delta|) \Delta^{2} d \Delta+O\left(\Delta^{4}\right)
\end{aligned}
$$

so that

$$
\mu_{1}(x)=\frac{1}{2} \frac{\partial \mu_{2}}{\partial x}+O\left(\Delta^{4}\right)
$$

and substituting (3.2) into (3.1) results in

$$
E_{e}(x) \frac{\partial X}{\partial t}=\frac{\partial}{\partial x}\left(\frac{\mu_{2}(x)}{2} \frac{\partial X}{\partial x}\right)
$$

a one-dimensional diffusion equation. The boundary conditions necessary to determine $X(x, t)$ uniquely are

$$
\left.\frac{\partial X}{\partial x}\right|_{x=0}=0 \quad \text { and }\left.\quad \frac{\partial X}{\partial x}\right|_{x=f}=0
$$

since a pathogen cannot have a negative number of antibodies and will not have more than the maximum of $f$ antibodies. We also require an initial condition which we 
define as $X(x, 0)=f_{1} \delta(x), x \geq 0$, where $\delta(x)$ is the Dirac delta function. Here we let there be an initial concentration of magnitude, $f_{1}$, on the boundary at $x=0$ and zero concentration for $x>0$. This specifies that there are no attached antibodies initially. When we solve the diffusion equation numerically we let

$$
X(x, 0)= \begin{cases}f_{1}, & 0 \leq x<1 \\ 0, & 1 \leq x \leq f\end{cases}
$$

We note that (3.3) can be written as

$$
E_{e}(x) \frac{\partial X}{\partial t}=\frac{\mu_{2}(x)}{2} \frac{\partial^{2} X}{\partial x^{2}}+\frac{1}{2} \frac{\partial \mu_{2}}{\partial x} \frac{\partial X}{\partial x}
$$

and thus there are two components indicating how the distribution will change with time, namely, $X$ will diffuse in the direction of least antibodies, and will be balanced by what the equilibrium should be according to the probability distribution that influences the moment, $\mu_{2}$.

\section{Probability distribution for change in the number of antibodies}

To solve the diffusion equation we require a form for $R\left(x, x^{\prime}\right)$. Consider the quantum version of antibody-pathogen interactions. In a time characteristic of the interaction of an antibody with an antigen, of duration $\delta_{t}$, a bound site may dissociate with probability $q$ or remain bound with probability $1-q$. Here $q$ is related to the antibody's dissociation constant, $k_{D}$. Also, an antibody may attach to an unbound site with probability $p$ or an unbound site may remain unbound with probability $1-p$. Here $p$ is related to the antigen-antibody association rate, $k_{A}$. Then, if $v(i)$ is an integer, it is straightforward to show that

$$
P(i, j)=\sum_{k=\max \{i-j, 0\}}^{\min \langle-j, i]}\left(\begin{array}{c}
v(i) \\
j-i+k
\end{array}\right)\left(\begin{array}{l}
i \\
k
\end{array}\right) q^{k}(1-q)^{i-k} p^{j-i+k}(1-p)^{v(i)-(j-i+k)},
$$

where $P(i, j)$ denotes the probability that a pathogen bound at $i$ sites becomes a pathogen bound at $j$ sites in one interaction time and $\sum_{j=0}^{N} P(i, j)=1$ as required. The function, $v(i)$, represents the valency of the pathogen that has $i$ bound sites. Hlavacek $e t$ al. define the valence of the $i$ th state, $v(i)$, to be the number of sites that are available for receptor binding on a ligand that is bound at $i$ sites averaged over all possible microscopic states of the ligand [5]. Steric effects of ligand-receptor binding can decrease the effective number of available sites. However, here we neglect steric effects and take the valence of the $i$ th state to be given by $v(i)=f-i$. 


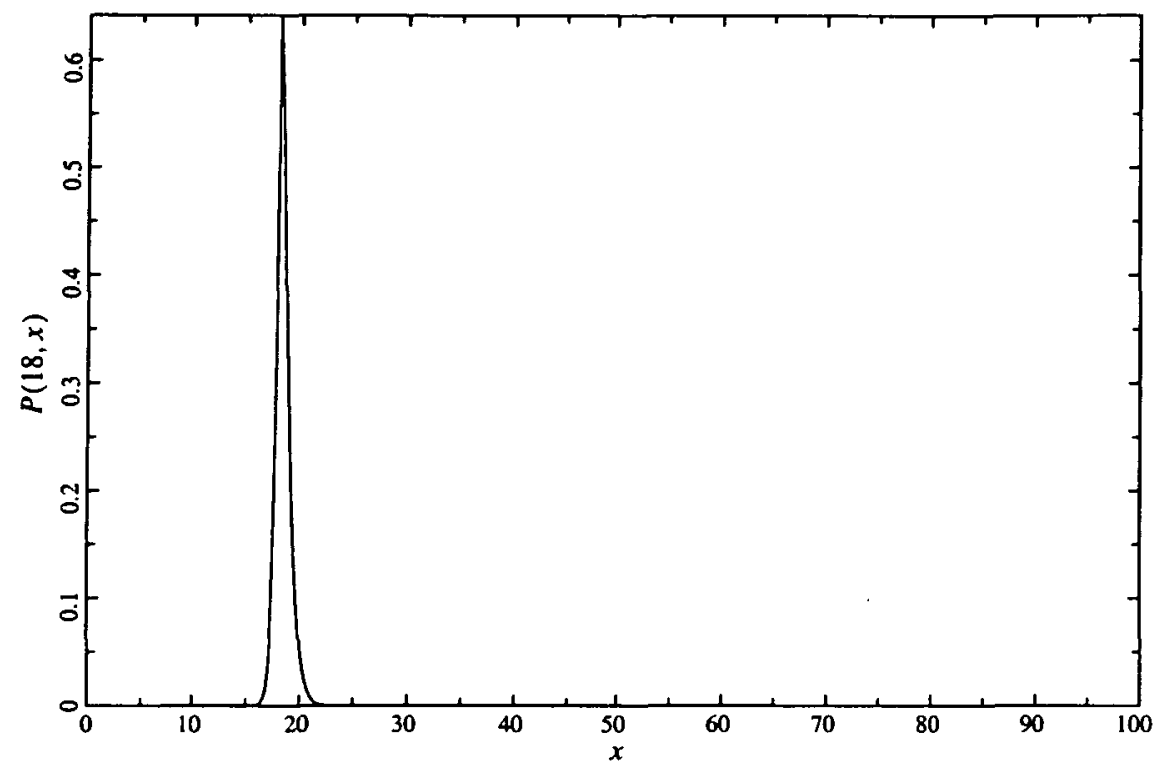

FIGURE 1. A typical probability distribution for moving from one antibody level to another. Here we illustrate the probability of a pathogen bound with 18 antibodies becoming a pathogen bound by $x$ antibodies in one interaction time. Here $p=0.005, q=0.003$ and $f=100$.

Since $\Gamma(n+1)=n$ !, we take the analogous probability distribution for the approximate continuous distribution, namely,

$$
P\left(x, x^{\prime}\right)=\int_{\max \left\{x-x^{\prime}, 0\right\}}^{\min \left(\zeta-x^{\prime}, x\right\}} C\left(x, x^{\prime}\right) q^{\zeta}(1-q)^{x-\zeta} p^{x^{\prime}-x+\zeta}(1-p)^{f-x^{\prime}-\zeta} d \zeta,
$$

where $C\left(x, x^{\prime}\right)$ is the number of ways a pathogen bound at $x$ sites can become a pathogen bound at $x^{\prime}$ sites, and can be expressed as

$$
C\left(x, x^{\prime}\right)=\frac{\Gamma(f-x+1) \Gamma(x+1)}{\Gamma\left(f-x^{\prime}-\zeta+1\right) \Gamma\left(x^{\prime}-x+\zeta+1\right) \Gamma(\zeta+1) \Gamma(x-\zeta+1)} .
$$

An example of the probability distribution is illustrated in Figure 1.

Then $k\left(x, x^{\prime}\right)=k_{1} P\left(x, x^{\prime}\right)$, where $k_{1}$ is a rate parameter that incorporates the interaction time $\delta_{t}$. The equilibrium distribution, $E_{e}(x)$, can now be determined by ensuring $P\left(x^{\prime}, x\right) E_{e}\left(x^{\prime}\right)=P\left(x, x^{\prime}\right) E_{e}(x)$, leading to

$$
E_{e}\left(x^{\prime}\right)=\frac{P\left(x, x^{\prime}\right)}{P\left(x^{\prime}, x\right)} E_{e}(x) .
$$

Integrating both sides of (4.1) over the interval $x^{\prime}=(0, f)$ and noting that because we are not considering any source or loss, the number of pathogens will remain at a 

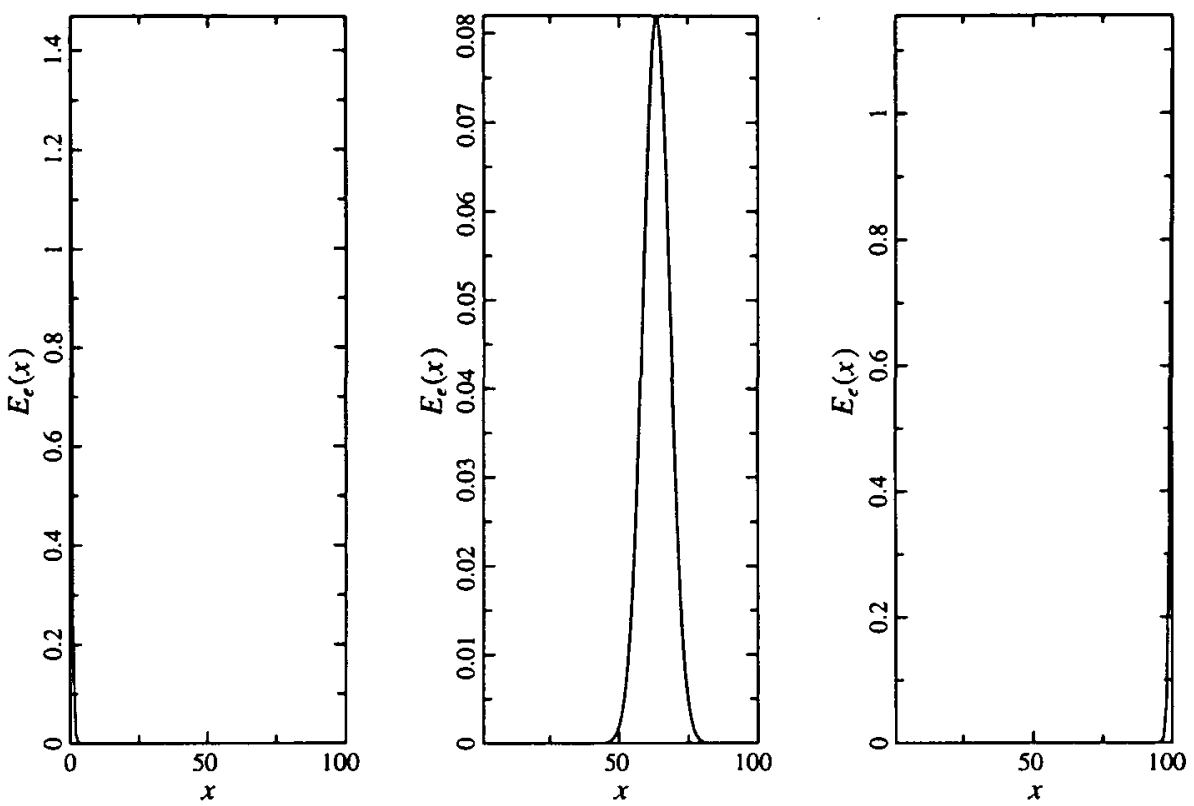

FIGURE 2. Plots of the equilibrium distribution, $E_{e}(x)$. We take $f_{1}=f=100$. (a) Binding probability low relative to dissociation probability ( $p=0.00005, q=0.03$ ). (b) Binding probability comparable to dissociation probability ( $p=0.005, q=0.003$ ). (c) Binding probability high relative to dissociation probability $(p=0.05, q=0.0003)$.

fixed level, $\int_{0}^{f} E_{e}\left(x^{\prime}\right) d x^{\prime}=E_{0}$, then we obtain

$$
E_{e}(x)=E_{0}\left[\int_{0}^{f} \frac{P\left(x, x^{\prime}\right)}{P\left(x^{\prime}, x\right)} d x^{\prime}\right]^{-1},
$$

which is illustrated in Figure 2 for various probabilities, $p$ and $q$.

In the limiting case as $p \rightarrow 0$ (that is, antibodies do not attach to unbound sites because the antibody and ligand site are not complementary), $E_{e}(x)=E_{0} \delta(x)$. Although the reaction of ligand-receptor binding is reversible, in particular cases of specific binding the dissociation reaction can be neglected [21]. Then in the limiting case as $q \rightarrow 0$ (that is, irreversible binding), $E_{e}(x)=E_{0} \delta(x-f)$.

\section{Numerical solution}

Given that we now have an expression for $R\left(x, x^{\prime}\right)$, an expression for the kernel of our diffusion equation can be determined. The magnitude of the kernel function varies considerably with the probabilities for binding and dissociation. This greatly 

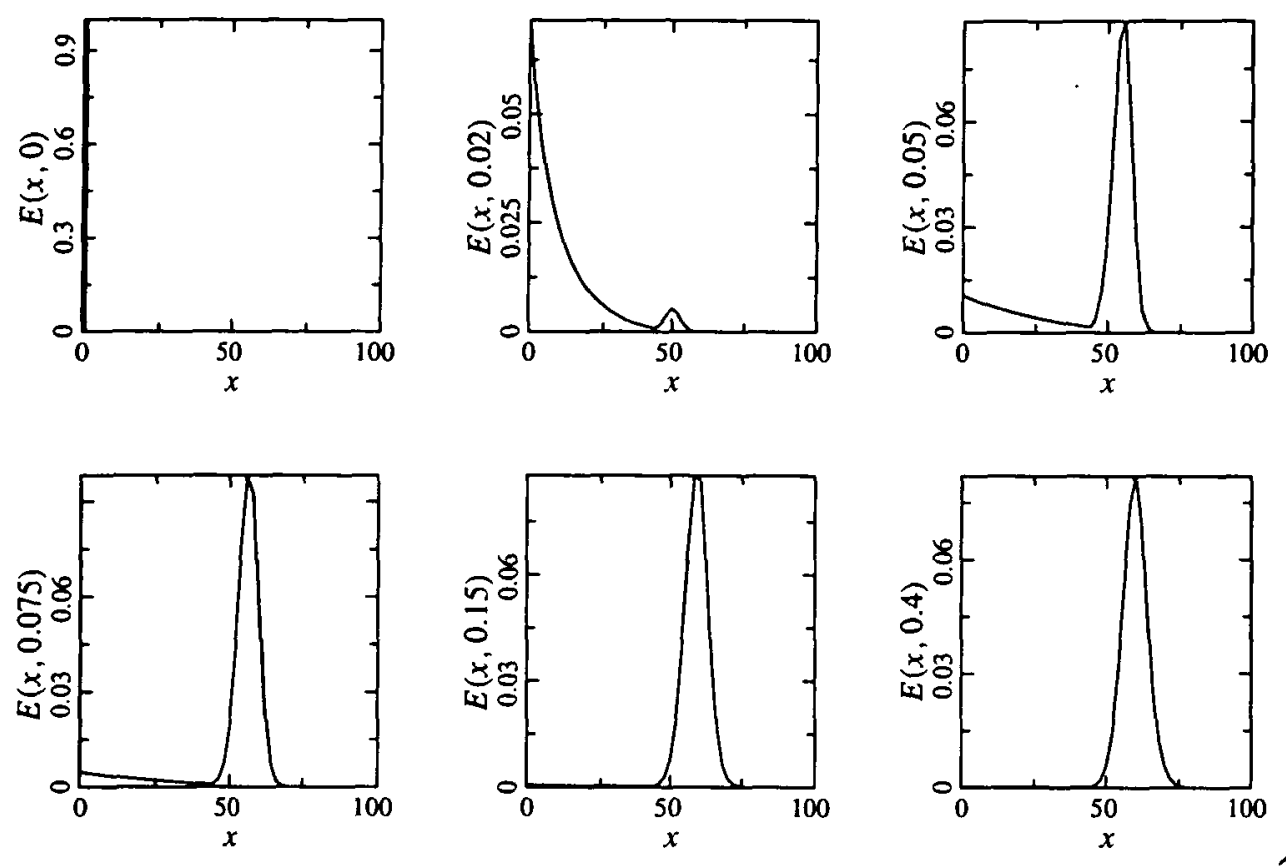

FIGURE 3. Sequence of numerical solutions for $E(x)$, the pathogen concentration of antibody distribution, for various times. Here $p=0.005$ and $q=0.003$.

influences the time for diffusion. We employ a fully implicit vertex-centred finite volume method [9] to obtain numerical solutions to the one-dimensional diffusion equation, (3.3), and then use (2.3) to revert to the solution for $E(x, t)$, the concentration of pathogens with $x$ antibodies attached at time $t$. Figures 3 and 4 illustrate the solutions for $E$ at various times, for two different expressions of the kernel, $\mu_{2}(x)$, corresponding to relative medium and large probabilities of antibody attachment. We do not display the solution for $E$ when the probability of antibody attachment is small because there is little change from the initial distribution.

\section{Discussion}

The humoral arm of the immune system is crucial in neutralising many infectious agents. The mechanisms by which humoral immunity is effective have been studied experimentally $[2,15,16]$ and theoretically $[13,17,18]$. Humoral immunity is mediated by $\mathbf{B}$ lymphocytes through the release of antibodies specific for attachment to a pathogen's binding sites. Increasing the level of antibodies attached to a pathogen's surface binding sites inhibits cell infection and increases pathogen clearance. The 

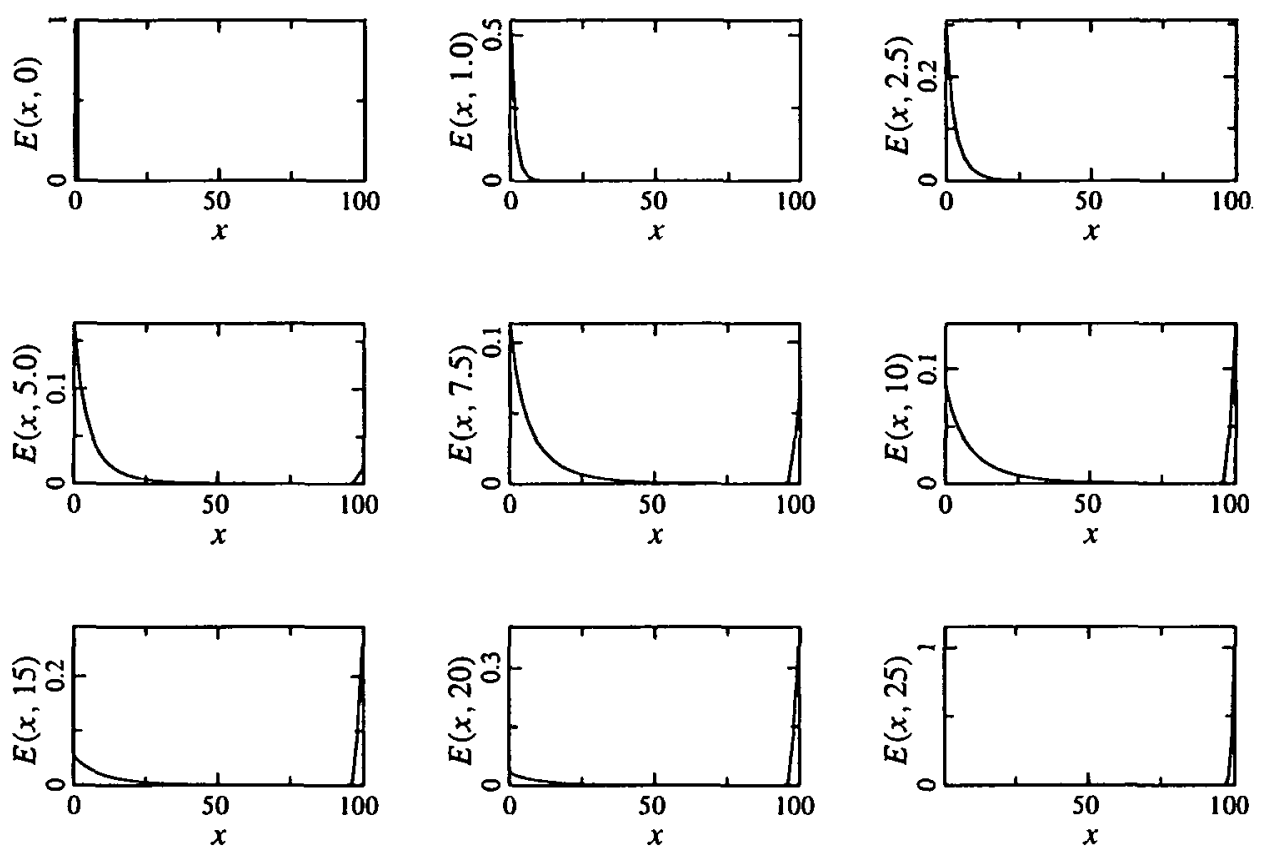

FIGURE 4. Sequence of numerical solutions for $E(x)$, the pathogen concentration of antibody distribution, for various times. Here $p=0.05$ and $q=0.0003$.

central theoretical problem associated with the binding of multivalent ligands is to predict the aggregate size distribution, from which all quantities of interest can be calculated [12]. When comparing theory with experiment it is often important to know this distribution because some types of cells respond differently to different size aggregates. For example, small numbers of $\operatorname{IgE}$ antibodies are relatively ineffective at stimulating degranulation of rat basophilic leukemia cells (RBL) cells, whereas larger aggregates are quite effective [4]. Human basophil cells also display large sensitivity to aggregate size [7]. However, predicting the aggregate size distribution requires the solution of a very large number of coupled ordinary differential equations. We have presented the master equation for pathogen-antibody levels in discrete and classical forms and shown how the classical master equation can be transformed to an equivalent diffusion equation in a non-dimensional variable. Thus we have reduced a system of $N$ ( $N$ usually very large) coupled ordinary differential equations to a diffusion equation. The diffusion equation is much easier to work with and the theory of such an equation is well known. We developed a probabilistically inferred rate of transition between antibody levels for the pathogen which influenced the non-constant coefficient of the diffusion equation. We determined equilibrium distributions and solved 
the one-dimensional diffusion equation numerically for solutions tending towards the equilibrium distributions. The theory may be generally applicable to any extracellular infectious agent.

\section{References}

[1] J. Bentz, S. Nir and D. G. Covell, "Mass action kinetics of virus-cell aggregation and fusion", Biophys. J. 54 (1988) 449-462.

[2] M. Cogne, "Humoral immunity. B lymphocytes; immunoglobulins (structure, diversity, function); clinical practice investigations; concept of humoral immunity deficiency", La Revue Du Praticien 51 (2001) 193-202.

[3] M. Dembo and B. Goldstein, "Theory of equilibrium binding of symmetric bivalent haptens to cell surface antibody: Application to histamine release from basophils", J. Immunol. 121 (1978) 345-353.

[4] C. Fewtrell and H. Metzger, "Larger oligomers of IgE are more effective than dimers in stimulating rat basophilic leukemia cells", J. Immunol. 125 (1980) 701-710.

[5] W. S. Hlavacek, R. G. Posner and A. S. Perelson, "Steric effects on multivalent ligand-receptor binding: Exclusion of ligand sites by bound cell surface receptors", Biophys. J. 76 (1999) 30313043.

[6] J. Keck and G. Carrier, "Diffusion theory of nonequilibrium dissociation and recombination", $J$. Chem. Phys. 43 (1965) 2284-2298.

[7] D. W. MacGlashan, S. P. Peters, J. Warner and L. M. Lichtenstein, "Characteristics of human basophil sulfide peptide leukotriene release: Releasability defined as the ability of the basophil to respond to dimeric cross-links", J. Immunol. 136 (1986) 2231-2239.

[8] C. S. McKee, "Detailed balancing", Applied Catalysis A: General 154 (1997) N2-N3.

[9] M. Ohlberger and C. Rohde, "Adaptive finite volume approximations for weakly coupled convection dominated parabolic systems", IMA J. Numerical Anal. 22 (2002) 253-280.

[10] A. S. Perelson, "A model for antibody mediated cell aggregation: rosette formation", in Mathematics and computers in biomedical applications (eds. J. Eisenfeld and C. DeLisi), (Elsevier, New York, 1985) 31-37.

[11] A. S. Perelson and C. DeLisi, "Receptor clustering on a cell surface. I. Theory of receptor crosslinking by ligands bearing two chemically identical functional groups", Math. Biosci. 49 (1980) 87-110.

[12] R. G. Posner, C. Wofsy and B. Goldstein, "The kinetics of bivalent ligand-bivalent receptor ggregation: ring formation and the breakdown of the equivalent site approximation", Math. Biosci. 126 (1995) 171-190.

[13] A. Rundell, R. DeCarlo, H. HogenEsch and P. Doerschuk, "The humoral immune response to haemophilus influenzae Type $b$ : A mathematical model based on $\mathrm{T}$-zone and germinal center B-cell dynamics", J. Theor. Biol. 194 (1998) 341-381.

[14] R. Schweitzer-Stenner, I. Licht, I. Luscher and I. Pecht, "Dimerization kinetics of the IgE-class antibodies by divalent haptens. II. The interaction between intact IgE and haptens", Biophys. J. 63 (1987) 563-568.

[15] M. K. Slifka, "Mechanisms of humoral immunity explored through studies of LCMV infection", Current Topics in Microbiology and Immunology 263 (2002) 67-81.

[16] M. K. Slifka, R. Antia, J. K. Whitmire and R. Ahmed, "Humoral immunity due to long-lived plasma cells", Immunity 8 (1998) 363-372. 
[17] O. A. Smirnova, "Study of cyclic kinetics of immunity by mathematical modeling methods", Kosmicheskaia Biologiia i Aviakosmicheskaia Meditsina 25 (1991) 53-56.

[18] I. Sobottka. F. Iglauer, T. Schuler, C. Schmetz, G. S. Visvesvara, H. Albrecht, D. A. Schwartz, N. J. Pienizzck, K. Bartscht, R. Laufs and J. Schottelius, "Acute and long-term humoral immunity following active immunization of rabbits with inactivated spores of various Encephalitozoon spccies", Parasitology Research 87 (2001) 1-6.

[19] P. L. Stewart, C. Y. Chiu, S. Huang, T. Muir, Y. Zhao, B. Chait, P. Mathias and G. R. Nemerow, "Cryo-EM visualization of an exposed RGD epitope on advenovirus that escapes antibody neutralization", EMBO J. 16 (1997) 1189-1198.

[20] H. Su, G. J. Spangrude and H. D. Caldwell, "Expression of Fc- $\gamma$ RIII on Hela 229 cells: possible effect on in vitro neutralization of chlamydia trachomatis", Infect. Immun. 59 (1991) 3811-3814.

[21] I. V. Surovtsev, I. A. Razumov, V. M. Nekrasov, A. N. Shvalov, J. T. Soini, V. P. Maltsev, A. K. Petrov, V. B. Loktcv and A. V. Chernyshev, "Mathematical modeling the kinetics of cell distribution in the process of ligand-receptor binding", J. Theor: Biol. 206 (2000) 407-417. 